\title{
The mechanical response of a syntactic polyurethane foam at low and high rates of strain
}

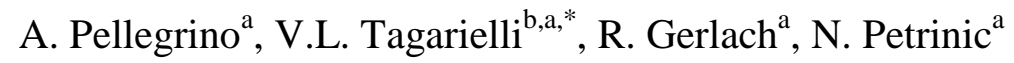 \\ (a) Department of Engineering Science, University of Oxford, Oxford OX1 3PJ \\ (b) Department of Aeronautics, Imperial College London, London SW7 2AZ
}

\begin{abstract}
Quasi-static and dynamic experiments are conducted to characterise the mechanical response of a syntactic foam comprising hollow glass microballoons in a polyurethane matrix. Stress versus strain histories are measured in uniaxial tension and compression as well as in pure shear, at strain rates ranging from $10^{-4}$ to $10^{3} \mathrm{~s}^{-1}$, via non-standard experimental techniques; quasi-static in-situ tests are conducted to visualise the deformation mechanisms in tension and compression. The material displays a pronounced sensitivity to the imposed strain rate and relatively high tensile and shear ductility at both low and high strain rates. A tension/compression asymmetry is displayed in quasi-static tests but is lost at high rates of strain.
\end{abstract}

Keywords: Syntactic Foam; Fracture; High Strain Rate;

Mechanical properties; Polyurethane

Revised submission to International Journal of Impact Engineering, July 2014

*Corresponding author; email: v.tagarielli@imperial.ac.uk. 


\section{INTRODUCTION}

Polymer matrix syntactic foams (PSF) are a class of lightweight materials that comprise thinwalled hollow particles (micro-balloons) dispersed in a polymeric matrix material [1],[2], offering lower density and, in certain cases, higher modulus than the pure matrix material [3]. Major advantages of PSF are their ease of manufacturing, their high compressive strength-toweight ratio and the possibility to tailor material properties by adjusting the size, wall thickness, volume-fraction as well as size distribution of the hollow particles. Typical areas of applications of PSF are within the marine, aerospace and ground transportation industries, where their low density and adjustable mechanical properties are of considerable advantage. PSF can be used as the core material in sandwich construction or to fill hollow lightweight structures in order to achieve mechanical damping and delay the onset of buckling instabilities.

The mechanical performance of PSF has recently received considerable interest from researchers, who have investigated the dependence of material properties on the microstructure ([3],[4]), the possibility of creating functionally graded foams ([5],[6]) and damage mechanisms on micro-balloons due to mechanical loading [7]. Most available studies on the mechanical response of PSF focused on their quasi-static compressive loading ([8],[9]) or compressive and tensile loading ([3],[10],[11]). Some research has focused on the high strain rate response in compression, and these studies reported significant strain rate sensitivity ([12-17]). Few or no studies investigated the tensile and shear response of relatively soft PSF at high rates of strain, due to the difficulties associated with these measurements [18-24].

On the other hand the tensile and shear response of these materials are substantially different from their compressive response, and this information is needed to inspire and motivate the development of accurate and effective constitutive models for these materials. In order to overcome this gap in the current literature, we employ low-impedence metallic Hopkinson bars to measure the dynamic material response in tension, compression and shear. Three different bespoke Hopkinson bar setups and corresponding specimen designs are employed in order to generate valid stress versus strain histories at high rates of strain. The dependence of the mechanical responses upon the imposed strain rates is analysed. 
Material and specimens are described in Section 2, while Section 3 presents the experimental techniques employed and the associated measurements. Results are discussed in Section 4.

\section{MATERIAL AND SPECIMENS}

The material under investigation is a syntactic foam comprising a polyurethane matrix and reinforcing hollow glass micro balloons (Eccosphere 311 SID) of external diameter ranging from $10-130 \mu \mathrm{m}$ and average wall thickness of $1.15 \mu \mathrm{m}$. The volume fraction of the reinforcing balloons (intended as the ratio of the volume occupied by the spheres and the total volume) was of approximately 0.5 and the overall material density was measured as $700 \mathrm{~kg} \mathrm{~m}^{-3}$. The foam was produced by Huntsman Advanced Materials ${ }^{\dagger}$ and was received in the form of thick plates of dimensions 200x200x50 mm. The foam was easy to machine and test specimens were extracted from these plates via conventional subtractive methods.

The specimens employed in tension and compression tests are shown in Fig. 1a. Compressive specimens were circular cylinders of diameter $4 \mathrm{~mm}$ and height $6 \mathrm{~mm}$. For the tensile tests, axisymmetric dogbone samples were manufactured, of gauge diameter $10 \mathrm{~mm}$ and gauge length $6 \mathrm{~mm}$. An integral M16 thread was machined at the end of these foam specimens to allow mechanical connection to the different test rigs employed in this study.

In order to measure the response in shear, torsion specimens were produced in the form of hollow, thin-walled circular cylinders as illustrated in Fig. 1b. The wall thickness of these cylinders was substantially reduced in the central portion in order to obtain a gauge section of uniform thickness; this had external diameter $50 \mathrm{~mm}$, wall thickness $2.5 \mathrm{~mm}$ and height $4 \mathrm{~mm}$. The specimen ends were then bonded using an epoxy-based adhesive to steel holders; these had the form of a thin plate with an integral hexagonal nut at the centre, produced via CNC milling. Holes were drilled in the flat, thin portion of the holders in order to reduce their mass.

Preliminary in-situ experiments were conducted in an environmental SEM by loading the material in compression and tension at quasi-static rates of strain. Cubes measuring

\footnotetext{
${ }^{\dagger}$ Ickleton Road, Duxford, Cambridge CB2 4QA, UK.
} 
$7 \times 7 \times 7 \mathrm{~mm}$ and small, flat dogbone specimens (of gauge section measuring $5 \times 2 \times 8 \mathrm{~mm}$ ) were used in the in-situ compression and tension test, respectively.

It merits comment that in both tension and compression, as well as torsion, tests at different strain rates were conducted on identical specimens, in order to allow a direct comparison of the quasi-static and dynamic responses.

\section{EXPERIMENTAL METHODS}

\subsection{Quasi-static in-situ experiments}

The specimens described above were polished on one face down to the finish given by a 4000-grit abrasive paper and placed in a miniature, screw-driven tension/compression loading stage (Deben Microtest M5000). The stage was placed in a Hitachi environmental SEM microscope and the cross-heads of the loading stage were displaced at a velocity such as to achieve a nominal strain rate of $10^{-3} \mathrm{~s}^{-1}$. Loading was interrupted occasionally in order to perform high resolution scans. Results of these tests are shown in Fig. 2; the figure illustrates the material microstructure (left-hand side), which comprised hollow spheres of different diameters; some surface damage, induced on the sample from the polishing procedure, is visible.

Figure 2 also presents SEM photographs taken at a macroscopic nominal strain of approximately $15 \%$ and $5 \%$ for the compression and tension tests, respectively. In the compressed sample we observe evidence of fracturing of the microballoons, by wing-cracks propagating in the direction of loading, as expected for a brittle material in compression; microcracks are also visible in the polymeric matrix at this strain, propagating in the direction of loading. Repeated experiments showed that fracture of the glass spheres initiated at compressive strains as low as $4 \%$ and at the spheres of larger diameter. In contrast, in the tensile sample we observe straight cracks developing in the glass spheres in a direction perpendicular to loading; matrix cracking is also observed, triggered by the stress concentration at a large defect (a missing microballoon). Additional experiments showed that fracture of the microspheres initiates at strains of the order of $2 \%$, again at the microballoons of larger diameter. 


\subsection{Quasi-static tests}

Compression and tension tests were conducted at low strain rates in a screw-driven Zwick tensometer; the applied load was recorded by a resistive load cell while a non-contact laser extensometer was employed to record the compressive and tensile strains. A high resolution camera was used to video-record the macroscopic deformation mechanisms and to obtain additional measurements of the axial strains via image analysis; these matched those recorded by the laser extensometer. Preliminary experiments, conducted on specimens oriented along three perpendicular directions, revealed that the material was isotropic.

Static torsion tests were performed on a bespoke apparatus consisting of two circular, Titanium torsion bars supported in a horizontal position and free to rotate about their axis; the bars, of length $2.4 \mathrm{~m}$ and diameter $25.4 \mathrm{~mm}$, were equipped with resistive strain gauges in order to measure the shear strains in the bars and deduce from these the applied torque, after a preliminary calibration. The hexagonal ends of the torsion specimens described in Section 2 mechanically engaged, via contact, with matching hexagonal groves machined at the end of the torsion bars. The free end of one of the bars was mechanically clamped while the opposite end of the bar system was driven to rotate at appropriate angular velocity by an electrical motor. The strains in the gauge portion of the sample were measured by analysing a highresolution video footage of the experiments via the commercial software GOM Aramis ${ }^{\star}$. It was found that the shear strain was initially uniform in the gauge portion of the specimen; failure was triggered by an initial localisation of shear deformation on a plane perpendicular to the torsion axis, see Fig. 3; such localisation coincided with the initiation of microcracks oriented along the direction of maximum principal strain (at 45 degrees on the torsion axis); these microcracks subsequently coalesced to form a macroscopic crack perpendicular to the torsion axis, bringing the specimen to catastrophic failure.

\subsection{Medium strain rate tests}

In order to achieve strain rats of the order of $10 \mathrm{~s}^{-1}$ in tension and torsion, a bespoke hydraulic loading system was used. The rig consisted of a lightweight piston capable of quickly reaching a constant velocity of magnitude up to $2 \mathrm{~ms}^{-1}$; details of this apparatus are described, for example, in [25],[26]. Compression and tension tests were conducted at medium strain rates; the load history was measured by piezo-electric load cells mounted in series with

\footnotetext{
${ }^{\ddagger}$ GOM UK Ltd., 14 The Cobalt Centre, Siskin Parkway East, Coventry, CV3 4PE, UK.
} 
instrumented rods. A digital high speed camera (Vision Research Phantom v7) was used to measure the elongation of the specimens during the tests. Torsion experiments were not conducted at this strain rate.

\subsection{High strain rate tests}

To achieve strain rates of order $10^{2}-10^{3} \mathrm{~s}^{-1}$, representative of impact loading, compression, tension and torsion test were conducted employing three different split-Hopkinson bars. For compression tests, a classical split-Hopkinson's pressure bar was used. To ensure accurate measurement of the relatively low compressive strains in the bars, high sensitivity Magnesium alloy bars were employed; the input and output bars had had diameter $12.7 \mathrm{~mm}$ and length of $1 \mathrm{~m}$, while the impactor was $0.5 \mathrm{~m}$ long. Further details on this test apparatus have been published in [22],[27], where the same setup was employed to measure the high strain rate compressive response of closed-cell polymer foams and balsa wood. Strain histories in the specimen were both calculated from the recorded strain gauge signals and measured via highspeed photography, employing an ultra-high-speed camera (Specialised Imaging - SIMX16), which was typically operated at $2 \cdot 10^{5} \mathrm{fps}$. Measurements and predictions differed by less than $5 \%$.

Measuring the high strain rate tensile response of cellular solids presents considerable technical difficulties: (i) dogbone samples have to be used, and these are relatively long, resulting in long times to achieve dynamic equilibrium; (ii) long tensile pulses are needed, and correspondingly long impactors, input and output bars, in order to achieve applied tensile strains sufficient to break the sample; (iii) the classical architecture of tensile Hopkinson's bars results, if long bars are employed, in considerable sagging which in turn induces spurious loading pulses; (iv) one-dimensional stress wave analysis cannot be used to determine accurately the strain history, due to the abrupt changes in the prismatic geometry of the system, such as mechanical fasteners and an inevitable dog-bone specimen geometry. All these difficulties were overcome in this study by using a novel SHTB design, the details of which have recently been published in [28]. In brief, this apparatus comprised a slotted impactor which was accelerated by a pulling rod, powered by a compressed air piston system; the impactor was free to slide on the input bar, supported by the same bar. The slotted cross- 
section allowed supporting the input bar with supports at a pitch of $300 \mathrm{~mm}$, in order to substantially reduce sagging.

The input bar was made from Titanium and had a solid circular cross-section of diameter $20 \mathrm{~mm}$ and length $2.7 \mathrm{~m}$; the output bar, made from steel, had same length and a hollow circular cross-section of internal and external diameters 13.5 and $18 \mathrm{~mm}$, respectively; M16 threads were machined at the bars' ends in order to engage these with the tensile dogbone specimens described above. A copper pulse shaper was used to provide a slowly ramped loading pulse and to facilitate dynamic equilibrium; details of this are given in [28]. Strain histories were again deduced from analysis of high-speed videos, produced via the ultra-highspeed camera and associated flash-light illumination. One-dimensional wave theory was also employed to calculate the tensile strain in the specimen; it was found that the measured and calculated strain versus time histories differed, in first approximation, by a multiplicative factor. The calculated strain history was adjusted to match the direct measurements.

Dynamic torsion tests were performed using a torsion Hopkinson-bar apparatus. This coincided with the apparatus described above and employed to conduct quasi-static torsion tests, with appropriate modifications. Details of this experimental technique can be found in [29],[30]. In the high strain rate tests, one of the torsion bars (input bar) was mechanically clamped at a distance of $1 \mathrm{~m}$ from the specimen's end, while the opposite end was forced to rotate by the desired angle by an hydraulic motor, thereby storing strain energy in the bar. The output torsion bar engaged with the test specimen but was otherwise free to rotate about its axis. To initiate the test the mechanical clamping was suddenly released by inducing fracture of the steel clamping bolt by an ad hoc mechanism; this released the stored strain energy in the form of a torsional wave of the desired amplitude, which propagated along the bar at the shear wave speed. The input wave was partially reflected at the specimens and partially transmitted into the output bar due to the jump in mechanical impedance. $120 \Omega$ resistance strain gauges were used to measure the stress waves in the two bars. A continuous time history of the engineering shear strain in the gauge portion of the specimens was determined by a combination of wave and image analysis, with the video footage obtained via high-speed photography $\left(10^{4} \mathrm{fps}\right)$. 
In order to assess the validity of the high strain rate test, it was checked that the specimens achieved dynamic force equilibrium during the experiments. Figure 4 presents time histories of the forces (or torques) measured in the input and output bars for the different loading cases. In compression, equilibrium was achieved at a time of the order of $100 \mu \mathrm{s}$, corresponding to a nominal compressive strain of order $2 \%$. In tension, equilibrium was achieved at around $200 \mu \mathrm{s}$, which corresponded to a nominal tensile strain of $2 \%$. In torsion, the applied torques initially differed substantially, due to the acceleration of the relatively heavy specimen holders and some slack in the mechanical coupling of specimen and loading bars; however the input and output torques equalise at a time of order $200 \mu \mathrm{s}$, which corresponds to a nominal

engineering shear strain $\varepsilon_{x y}=\gamma_{x y} / 2=0.06$. We conclude that the measured dynamic stress/strain histories are valid at strains exceeding those reported above for each type of test; in this regime the strain rate was approximately constant for all loading cases.

\section{RESULTS AND DISCUSSION}

We proceed to present the stress versus strain histories measured in the experiments, to deduce from these the strain rate sensitivity of the material, and to construct failure envelopes in stress space. At all imposed strain rates, each type of test was repeated several times in order to analyse the scatter of the data. A summary of the measured material strength and applied strain rate in different loading conditions is shown in Table 1.

The gauge portions of the specimens employed in the compression, tension and torsion tests have different volumes, ranging from 75 to $7100 \mathrm{~mm}^{3}$. In order to exclude the possibility of a size-dependence of the mechanical response of the material, a preliminary set of quasi-static tension and compression experiments were conducted on specimens of identical geometry and different volumes, in the range $10-5000 \mathrm{~mm}^{3}$ and $200-6000 \mathrm{~mm}^{3}$ for compression and tension specimens, respectively. Experiments were conducted with cross-head velocities chosen to obtain a strain rate of order $10^{-4} \mathrm{~s}^{-1}$ in all tests, irrespective of specimen size. It was found that both the tensile and compressive responses were insensitive to specimen size. Dynamic SPHB compression tests were also conducted on cylindrical specimens of diameter 4 to $10 \mathrm{~mm}$; no dependence of the dynamic response upon specimen diameter was observed. 


\subsection{Quasi-static response}

Representative measured nominal stress versus strain histories recorded in quasi-static experiments are presented in Fig. 5; preliminary loading-unloading compression experiments at low strains revealed the viscoelastic nature of the foam, manifesting in a relatively large hysteresis. In all loading conditions, the material displays a highly non-linear response, with no clear transition between viscoelastic and inelastic behaviour; this is explained by the mechanisms of deformation observed in the in-situ tests, where microscopic damage mechanisms were observed to be triggered at strains as low as $2 \%$ for tension and $4 \%$ in compression.

In compression, the material displayed the classical response of a cellular solid, with a nonlinear loading phase and a stress peak followed by a plateau collapse phase, of approximately constant flow stress, and subsequent densification; macroscopic damage, in the form of a macroscopic crack propagating in the direction of loading, was observed at compressive strains in the range $0.55-0.65$. In tension, the response possessed a higher non-linearity compared with the compression tests, and collapse was reached at substantially lower stress than that observed in compression; again, this is in line with the microscopic observation that damage mechanisms in tension were triggered at lower strains than in compression; the flow stress reached a peak value before catastrophic fracture of the specimen was observed at applied tensile strains of the order of 0.2 . We note that this tensile ductility is very high compared with that observed for other cellular solids [31],[32]. At a strain rate of $10^{-4} \mathrm{~s}^{-1}$ the material stiffness was of order $400 \mathrm{MPa}$ in both tension and compression, while the initial Poisson ratio was 0.37 . The response in shear was similar to that observed in tension, with a slightly higher ductility.

\subsection{Sensitivity to the imposed strain rate}

We proceed to present the dependence of the measured stress/strain responses upon the imposed strain rate, see Fig. 6. The stress versus strain histories obtained from the Hopkinson bar experiments are plotted beyond the strain at which dynamic equilibrium was achieved in the tests. In general the foam displays only a mild sensitivity to strain rate in the range $\dot{\varepsilon}<10 \mathrm{~s}^{-1}$, while the elevation of flow stress with the applied strain rate is substantially more pronounced in the range $10 \mathrm{~s}^{-1}<\dot{\varepsilon}<10^{3} \mathrm{~s}^{-1}$. The measured increase in flow stress when the 
strain rate is increased from $10^{-3} \mathrm{~s}^{-1}$ to $10^{3} \mathrm{~s}^{-1}$ is superior to that reported by other authors for solid polyurethane (PU) [33] and PU foams [34]. In compression, the increase in flow stress for solid PU is of less than three times [33], but for PU foams it is of only $22 \%$ [34], for a foam of similar density as that tested here; for the material under investigation we find that the corresponding increase in compressive flow stress is of four times. We deduce that the additional rate sensitivity is induced by the presence of the glass spheres and the deformation mechanisms that this triggers. The increase in compressive stress measured for the PSF under investigation is also superior to that reported by other authors (e.g. [13]) for different syntactic foams of similar densities; these authors report an increase in flow stress of 2.5 times when the strain rate is increased from $10^{-3} \mathrm{~s}^{-1}$ to $10^{3} \mathrm{~s}^{-1}$.

The compressive collapse stress increases from around $10 \mathrm{MPa}$ in quasi-static tests to around $40 \mathrm{MPa}$ at $10^{3} \mathrm{~s}^{-1}$; the initial peak in compressive stress is observed at progressively lower strains as the strain rate is increased. Rate sensitivity is even more pronounced in tension experiments, with the peak tensile stress displaying a six-fold increase as the strain rate is varied from quasi-static rates to $10^{3} \mathrm{~s}^{-1}$; we note that the measured dynamic tensile strength is similar to the dynamic compressive strength, in contrast to what observed at low strain rate. Correspondingly, the tensile ductility decreases from 20 to $8 \%$ at high strain rate. In shear, the strain rate sensitivity is comprised between those observed in compression and tension. A summary of the dependence of the flow stress upon strain rate is given in Fig. 7. We note that at low strain rates the compressive response is much more repeatable than the tensile and shear responses; at high strain rates, the scatter in the measured compressive, tensile and shear strength is similar. This is not associated with the experimental techniques used but it is rather a feature of the material's response, suggesting that the microscopic failure mechanisms triggered at high strain rates are different from the quasi-static ones presented in Fig. 2.

\subsection{Failure envelope}

It is instructive to plot the measured failure stress (intended as the compressive collapse stress, or the peak flow stress in the case of tension and shear) in deviatoric versus hydrostatic stress space, and to observe the evolution of the failure envelope as the strain rate increases from low to high; this information is presented in Fig. 8. The figure contains averages of only a 
subset of the data in Fig. 7, namely the quasi-static tests conducted at $\dot{\varepsilon}=10^{-3} \mathrm{~s}^{-1}$ and the high-rate tests at strain rate in the range $200 \mathrm{~s}^{-1}<\dot{\varepsilon}<350 \mathrm{~s}^{-1}$.

At low strain rates the material displays a pronounced tension/compression asymmetry, with the uniaxial compressive strength exceeding the tensile one. It is interesting to note that at high strain rates such asymmetry is lost, with similar compressive and tensile strengths. We proceed to observe that the failure envelope measured at quasi-static strain rate is of nonconvex shape, which is theoretically admissible but unusual. The relatively low strength in quasi-static shear could be ascribed to the microscopic mechanisms of failure of the microballoons shown in Fig. 2. In a state of pure shear the material is loaded along two perpendicular directions by principal stresses equal in modulus but of opposite sign, $\sigma_{\mathrm{I}}=-\sigma_{\mathrm{II}}$. With the microscopic failure mechanisms observed in uniaxial tension and compression in mind, it appears likely that loading by two equal and opposite stresses in perpendicular directions should result in earlier failure of the microballoons, compared to the case of uniaxial loading. Different failure mechanisms are expected to be activated at high rates of strains, which result in a relatively stronger macroscopic shear response compared to the uniaxial tension and compression cases.

\section{CONCLUSION}

We conducted a comprehensive test campaign aimed at characterising the mechanical response of a polyurethane-based syntactic foam and at motivating theoretical constitutive models. We presented measurements of the tensile, compressive and shear response of the foam at strain rates ranging from quasi-static to $10^{3} \mathrm{~s}^{-1}$, as well as quasi-static in-situ experiments; no similar set of data had been previously published due to the difficulties associated with dynamic experiments, which were overcome by using bespoke Hopkinson bar setups and appropriate specimen designs. Tests at different strain rates were conducted on identical specimens to allow a direct comparison of the static and dynamic responses.

The main conclusions of the study are:

- The PU syntactic foam displays a strain rate sensitivity superior to that of solid PU and of conventional PU foams of similar density, indicating that the embedded glass 
microballoons trigger deformation mechanisms which enhance the response at high strain rate.

- The foam displays great tensile and shear ductility at low strain rates and partially retains this at high strain rates, making it an appealing core material for blast- and impact-resistant sandwich constructions.

- The foam displays a tension/compression asymmetry in the quasi-static regime but this is lost at high rates of strain. The material possesses higher rate sensitivity in tension than in compression.

\section{ACKNOLEDGEMENTS}

The authors would like to thank Dr J.A. Rongong of the University of Sheffield for enlightening conversations and useful information. Dr Pellegrino is grateful to the University of Catania (Italy) for the financial support of his internship at the Impact Engineering Laboratory of the University of Oxford and to GOM UK ltd for their assistance with the Aramis software. Dr Tagarielli acknowledges the help of Mr J. Chew and Mr S Pindoria (Imperial College London) with the in-situ experiments and the assistance of $\mathrm{Dr} \mathrm{K}$. Dragnevski and Mr S. Carter (University of Oxford) with sample preparation and experimental setup. 


\section{REFERENCES}

[ 1 ] NIELSEN, L. E. 1983. Elastic modulus of syntactic foams. Journal of Polymer Science Part B-Polymer Physics, 21, 1567-1568.

[ 2 ] NARKIS, M., KENIG, S. \& PUTERMAN, M. 1984. 3-PHASE SYNTACTIC FOAMS. Polymer Composites, 5, 159-165.

[ 3 ] GUPTA, N., YE, R. \& PORFIRI, M. 2010. Comparison of tensile and compressive characteristics of vinyl ester/glass microballoon syntactic foams. Composites Part BEngineering, 41, 236-245

[ 4 ] WOUTERSON, E. M., BOEY, F. Y. C., HU, X. \& WONG, S. C. 2005. Specific properties and fracture toughness of syntactic foam: Effect of foam microstructures. Composites Science and Technology, 65, 1840-1850.

[ 5 ] GUPTA, N. \& RICCI, W. 2006. Comparison of compressive properties of layered syntactic foams having gradient in microballoon volume fraction and wall thickness. Materials Science and Engineering a-Structural Materials Properties Microstructure and Processing, 427, 331-342.

[ 6] KISHORE, SHANKAR, R. \& SANKARAN, S. 2005. Gradient syntactic foams: Tensile strength, modulus and fractographic features. Materials Science and Engineering aStructural Materials Properties Microstructure and Processing, 412, 153-158.

[ 7 ] J., MAIRE, E., GIMENEZ, N. \& SAUVANT-MOYNOT, V. 2007. Experimental study of the compression behaviour of syntactic foams by in situ X-ray tomography. Acta Materialia, 55, 1667-1679.

[ 8 ] GUPTA, N., KISHORE, WOLDESENBET, E. \& SANKARAN, S. 2001. Studies on compressive failure features in syntactic foam material. Journal of Materials Science, 36, 4485-4491.

[ 9] SWETHA, C. \& KUMAR, R. 2011. Quasi-static uni-axial compression behaviour of hollow glass microspheres/epoxy based syntactic foams. Materials \& Design, 32, 4152-4163. [ 10 ] RIZZI, E., PAPA, E. \& CORIGLIANO, A. 2000. Mechanical behavior of a syntactic foam: experiments and modeling. International Journal of Solids and Structures, 37, 57735794.

[ 11] GUPTA, N. \& NAGORNY, R. 2006. Tensile properties of glass microballoon-epoxy resin syntactic foams. Journal of Applied Polymer Science, 102, 1254-1261. 
[ 12 ] SHUNMUGASAMY, V. C., GUPTA, N., NGUYEN, N. Q. \& COELHO, P. G. 2010. Strain rate dependence of damage evolution in syntactic foams. Materials Science and Engineering a-Structural Materials Properties Microstructure and Processing, 527, 6166-6177. [ 13 ] GUPTA, N. \& SHUNMUGASAMY, V. C. 2011. High strain rate compressive response of syntactic foams: Trends in mechanical properties and failure mechanisms. Materials Science and Engineering a-Structural Materials Properties Microstructure and Processing, 528, 7596-7605.

[ 14 ] KIM, H. S. \& OH, H. H. 2000. Manufacturing and impact behavior of syntactic foam. Journal of Applied Polymer Science, 76, 1324-1328.

[ 15 ] SONG, B., CHEN, W. W. \& LU, W. Y. 2007. Mechanical characterization at intermediate strain rates for rate effects on an epoxy syntactic foam. International Journal of Mechanical Sciences, 49, 1336-1343.

[ 16 ] LI, P., PETRINIC, N., SIVIOUR, C. R., FROUD, R. \& REED, J. M. 2009. Strain rate dependent compressive properties of glass microballoon epoxy syntactic foams. Materials Science and Engineering a-Structural Materials Properties Microstructure and Processing, $515,19-25$.

[ 17 ] SONG, B. \& CHEN, W. 2004. Dynamic compressive response and failure behavior of an epoxy syntactic foam. Journal of Composite Materials, 38, 915-936.

[18] GRAY GT, BLUMENTHAL WR. Split-Hopkinson pressure bar testing of soft materials, ASM Handbook. Mechanical Testing and Evaluation 2000;8:488-96.

[19] ZHAO H, GARY G, KLEPACZKO JR. On the use of a viscoelastic split Hopkinson pressure bar. International Journal of Impact Engineering 1997;19:319-30.

[20] CHEN W, ZHANG B, WU MJ. A split Hopkinson bar technique for low impedance materials. Experimental Mechanics 1999;39:81-5.

[21] SHARMA A, SHUKLA A, PROSSER RA. Mechanical characterization of soft materials using high speed photography and split Hopkinson pressure bar technique. Journal of Materials Science 2002;37:1005-17.

[22 ] AREZOO, S., TAGARIELLI, V.T, SIVIOUR, N., 2012, Compressive deformation of Rohacell foams: effects of strain rate and temperature, Internation Journal of Impact Engineering, Volume 51, January 2013, Pages 50-57.

[23] ZHAO H, G. GARY. A three dimensional analytical solution of the longitudinal wave propagation in an infinite linear viscoelastic cylindrical bar. Application to experimental 
techniques, Journal of the Mechanics and Physics of Solids Volume 43, Issue 8, August 1995, Pages 1335-1348.

[24] GRAY III, G. T., IDAR, D. J., BLUMENTHAL, W. R., CADY, C. M., and PETERSON, P. D. (1998). "High and low-strain rate compression properties of several energetic material composites as a function of strain rate and temperature." Proc., 11th International Detonation Symposium, Snowmass, Colorado. 76-84.

[25] HARDING, J. \& WELSH, L. M. 1983. A Tensile Testing Technique for FiberReinforced Composites at Impact Rates of Strain. Journal of Materials Science, 18, 18101826.

[26] GERLACH, R., SIVIOUR, C. R., PETRINIC, N. \& WIEGAND, J. 2008.

Experimental characterisation and constitutive modelling of RTM-6 resin under impact loading. Polymer, 49, 2728-2737.

[27] TAGARIELLI, V.L., DESPHANDE V.S, FLECK N.A. 2007 The high strain rate response of PVC foams and end-grain balsa wood. Composites: Part B 39 (2008) 83-91 [28] GERLACH, R., KETTENBEIL, C. \& PETRINIC, N. 2012. A new split Hopkinson tensile bar design. International Journal of Impact Engineering, 50, 63-67.

[29] LEWIS J.L. and CAMPBELL, J.D., The Development and Use of a Torsional Hopkinson Bar Apparatus, Exp. Mech., Vol 12 (No. 11), 1972, p 520-524

[30] GILAT, A. 2000 . Torsional Kolsky bar testing. In: Kuhn H, Medlin D, editors. ASM handbook: mechanical testing and evaluation, vol. 8. Materials Park, $\mathrm{OH}$ : ASM International, p. $505-15$

[31] AREZOO, S., TAGARELLI, V. L., PETRINIC N., and REED, J. M., 2011. The mechanical response of Rohacell foams at different length scales. J. Mater. Sci. 46(21):68636870.doi:10.1007/s10853-011-5649-7

[32] SIEGKAS, P., TAGARIELLI, V.L., PETRINIC, N., and LEFEBVRE, L.-P., 2011. The compressive response of a titanium foam at low and high strain rates. J. Of Mat.Sci., 46(8):2741-2747.

[33] SARVA, S.S., DESCHANEL, S., BOYCE, M.C., and CHEN, W., 2007. Stress-strain behavior of a polyurea and a polyurethane from low to high strain rates. Polymer, 48; 2208 2213.

[34] SUBHASH, G., LIU, Q., and GAO, X-L, 2006. Quasistatic and high strain rate uniaxial compressive response of polymeric structural foams. Int.J.Impact Eng., 32(7): 11131126. 
(a)
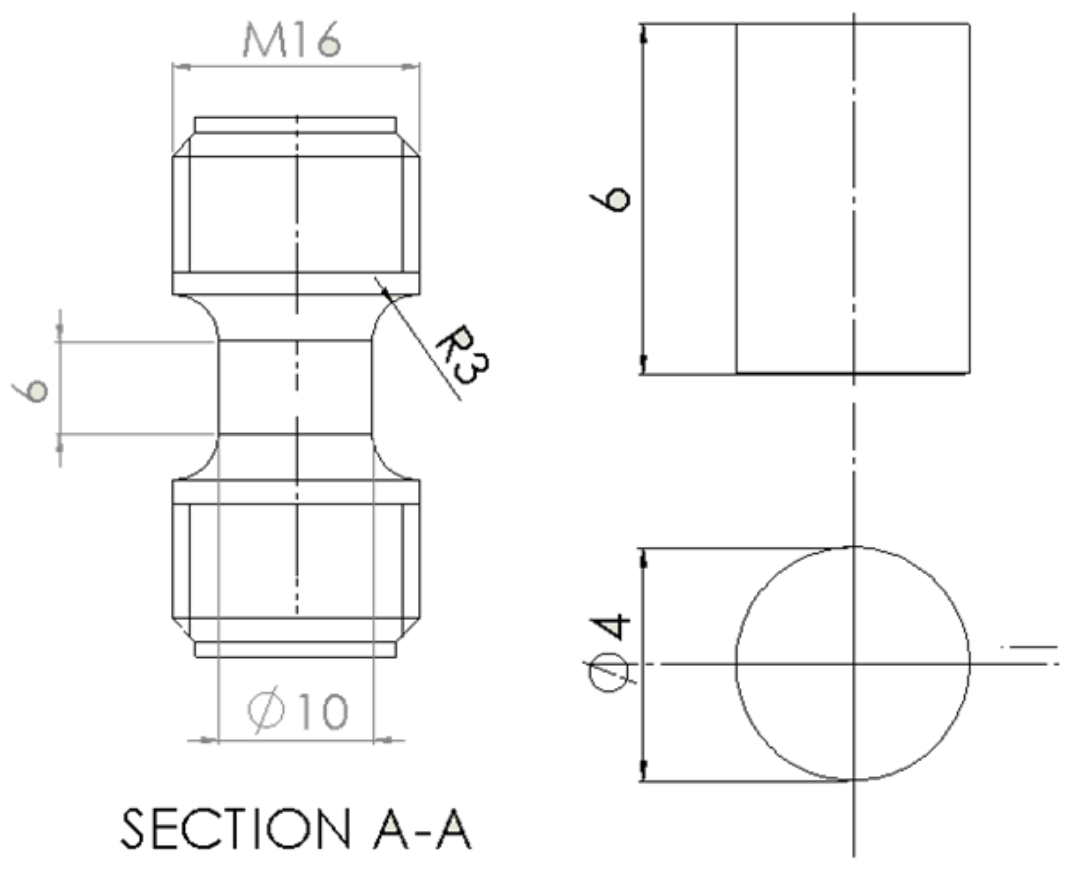

(b)

SECTION A-A

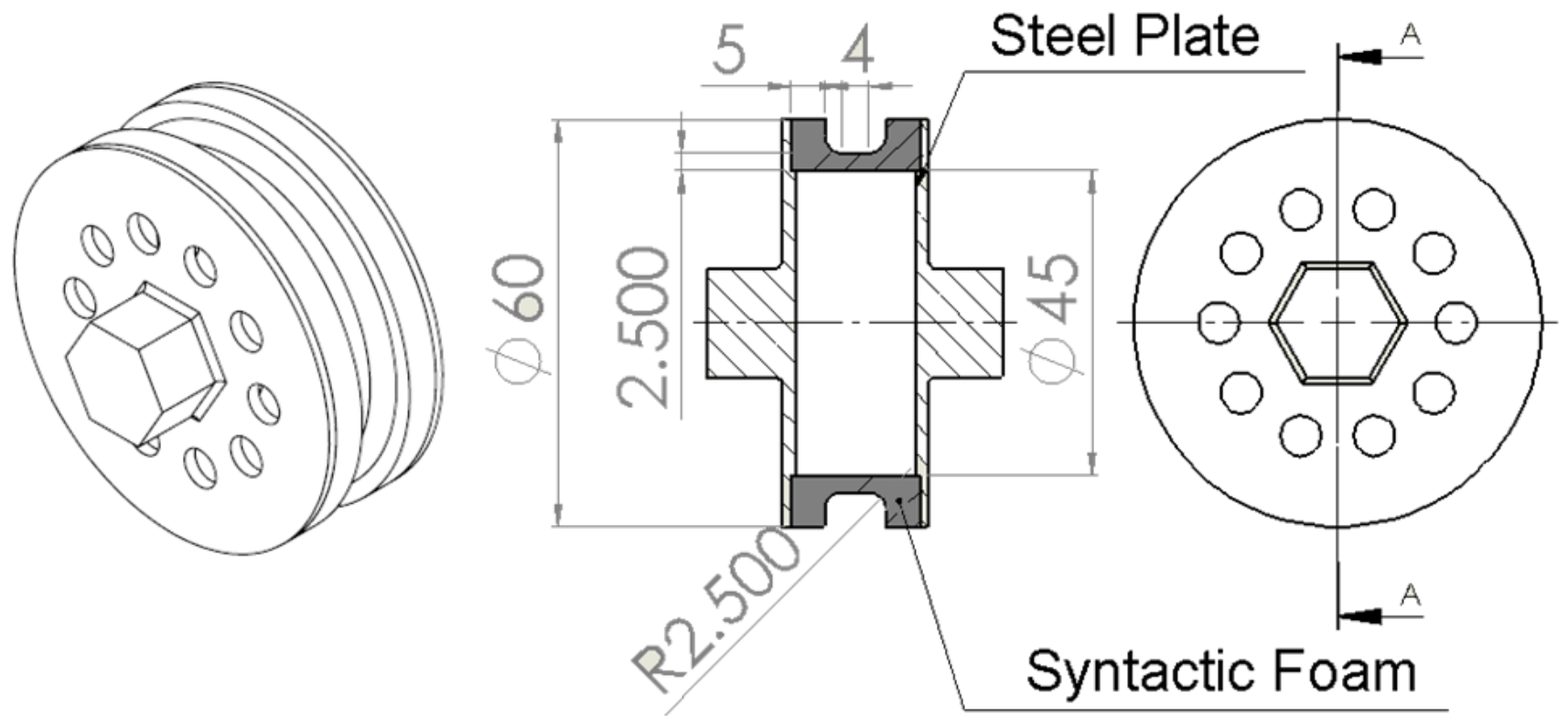

Fig 1. Geometry and dimensions of the foam specimens employed in this study in (a) the tension and compression experiments and (b) torsion experiments. 

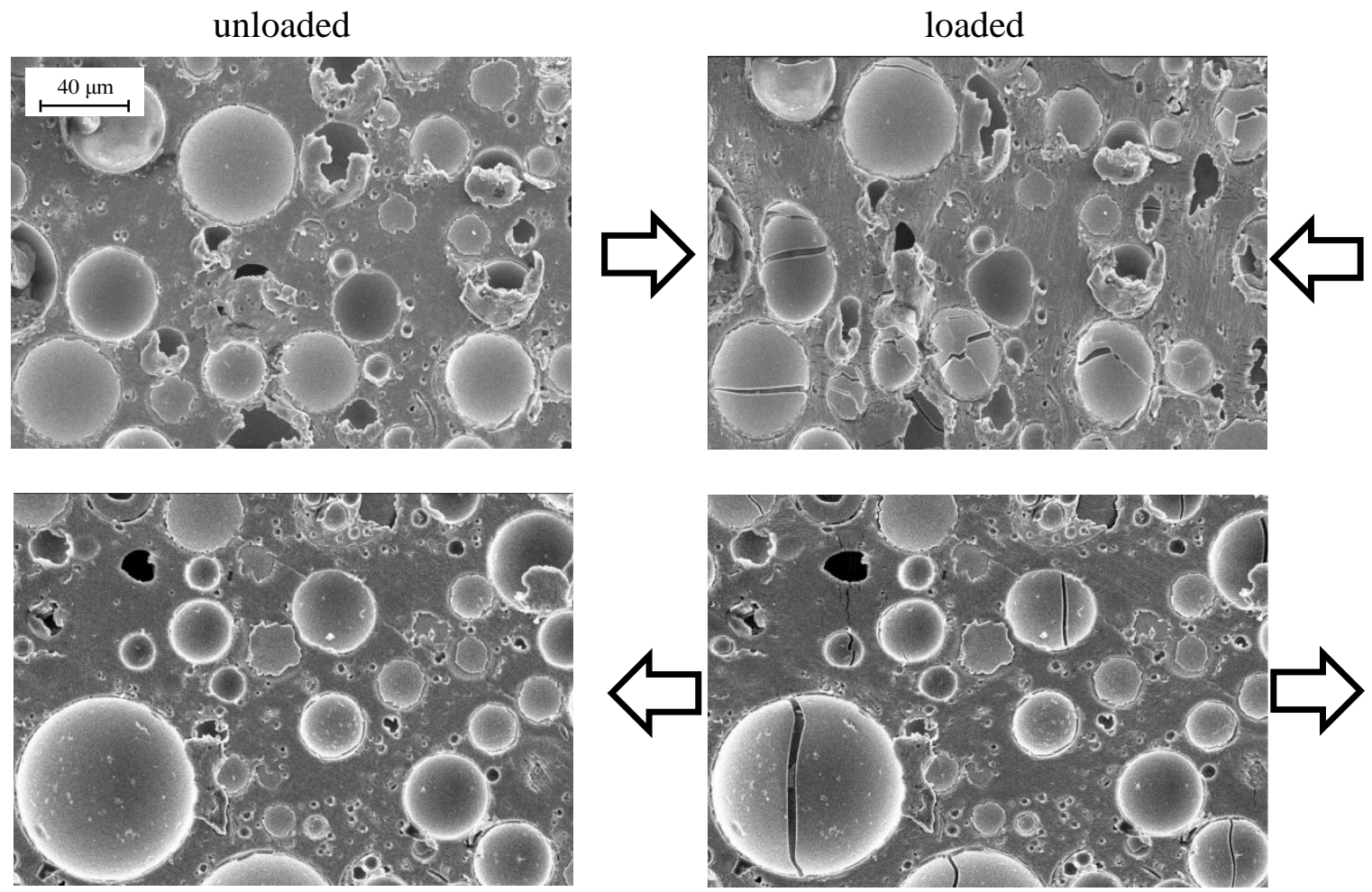

Fig 2. SEM images illustrating the microstructure of the foam and the microscopic mechanisms of deformation and fracture in quasi-static compression (top, $\varepsilon_{c} \approx 15 \%$ ) and tension (bottom, $\varepsilon_{t} \approx 5 \%$ ). 


\begin{tabular}{|c|c|c|c|}
\hline \multirow{3}{*}{\begin{tabular}{c} 
type oftest \\
\multirow{3}{*}{ tension }
\end{tabular}} & repetitions & average strain rate, $\mathrm{s}^{-1}$ & average collapse stress, MPa $^{*}$ \\
\cline { 2 - 4 } & 3 & 0.00015 & 7.46 \\
\cline { 2 - 4 } & 12 & 0.001 & 5.79 \\
\cline { 2 - 4 } & 4 & 12.5 & 9.55 \\
\hline \multirow{4}{*}{ compression } & 15 & 218 & 33.5 \\
\cline { 2 - 4 } & 2 & 0.00015 & 12.625 \\
\cline { 2 - 4 } & 12 & 0.0001 & 10.38 \\
\cline { 2 - 4 } & 2 & 0.001 & 10.66 \\
\cline { 2 - 4 } & 4 & 0.01 & 13.735 \\
\cline { 2 - 4 } & 6 & 7.855 & 14.01 \\
\cline { 2 - 4 } & 7 & 361 & 36.95 \\
\hline \multirow{3}{*}{ shear } & 3 & 1185 & 35.14 \\
\cline { 2 - 4 } & 4 & 0.00015 & 2.72 \\
\hline
\end{tabular}

Table 1. Summary of the experiments conducted in this study. 

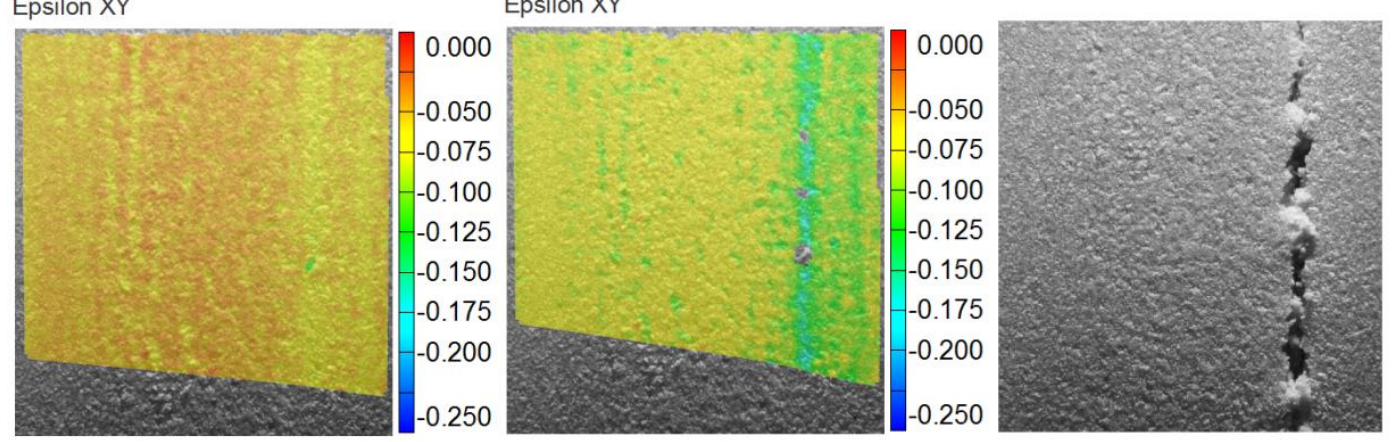

Fig 3 - Detail of the strain field observed during quasi-static torsion tests; the horizontal direction coincides with the torsion axis, and contours of the shear strains are showed at engineering shear strains of 0.05 and 0.075 , indicating an initial localisation of the shear deformation; a macroscopic crack is shown after final fracture of the specimen at a location corresponding to the initial localisation. The size of photographs is $4 \times 4 \mathrm{~mm}$. 

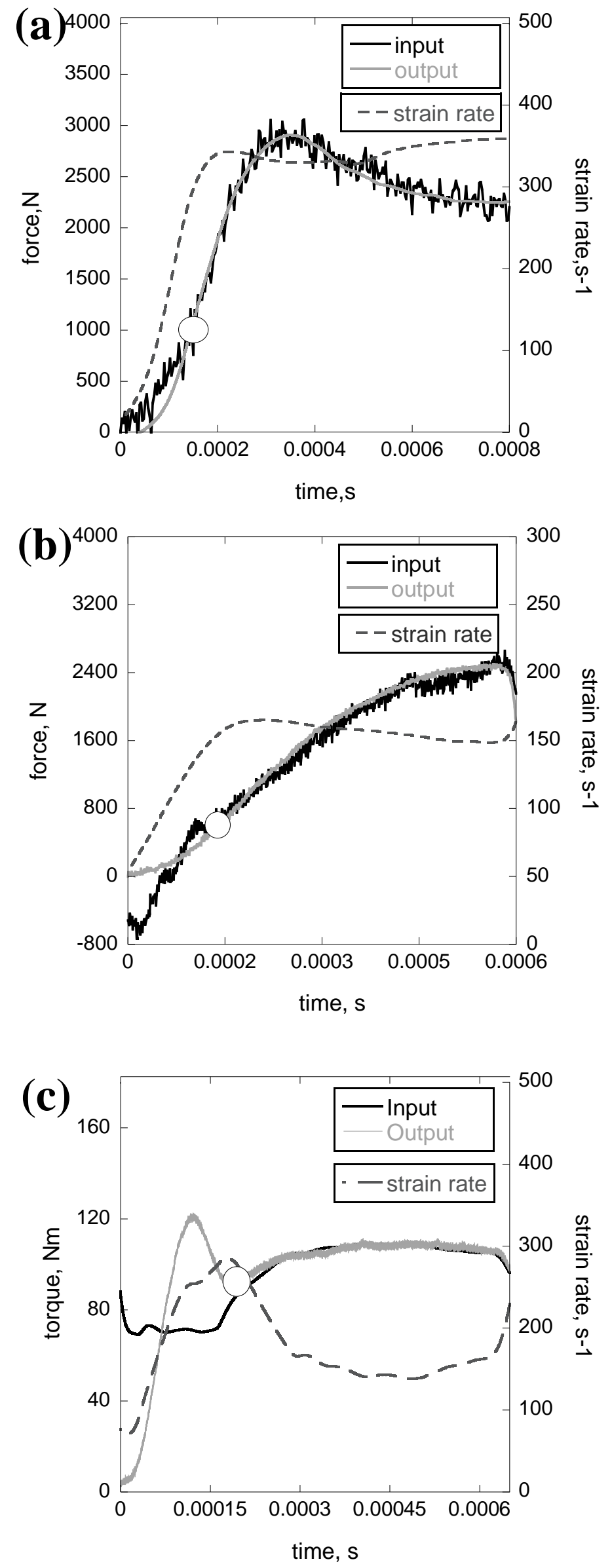

Fig 4. Representaive measured forces and torques in the input and output bars for $(\mathrm{a}, \mathrm{b})$ compression, $(\mathrm{c}, \mathrm{d})$ tension and $(\mathrm{e}, \mathrm{f})$ torsion tests at high strain rate. Dynamic equilibrium was achieved in all experiments. 


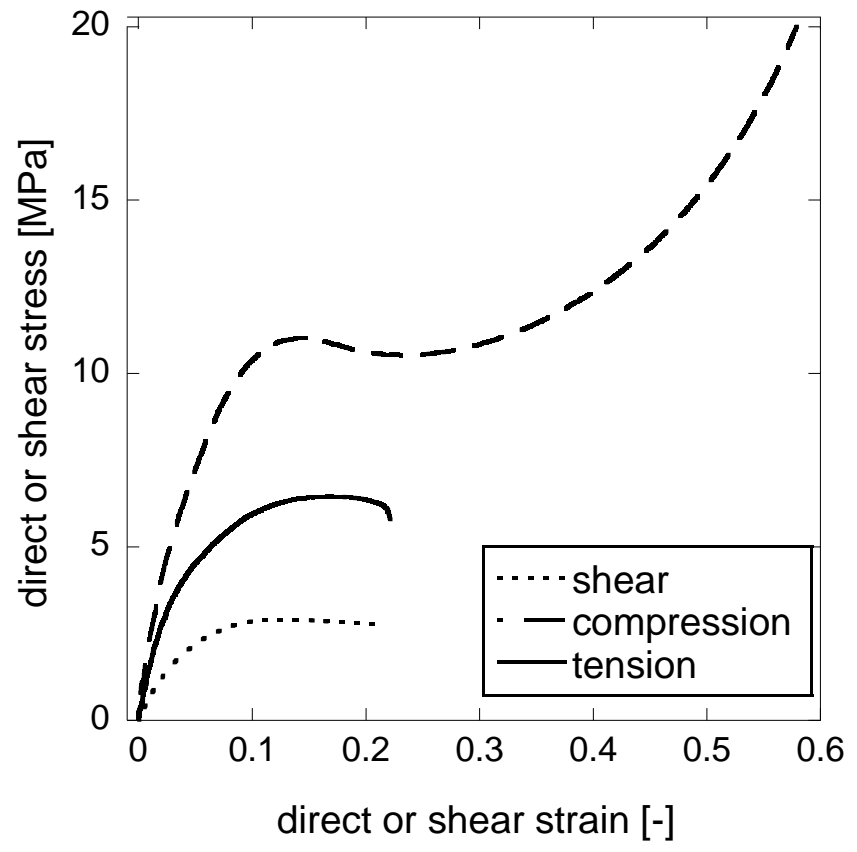

Fig. 5. Quasi-static compressive, tensile and shear responses in terms of nominal stress versus strain histories. 
(a)

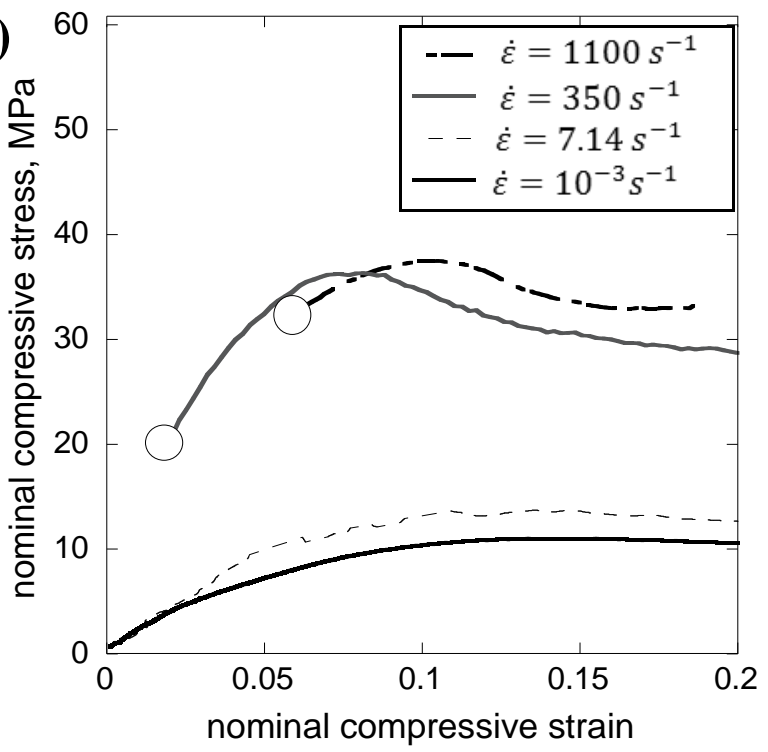

(b)

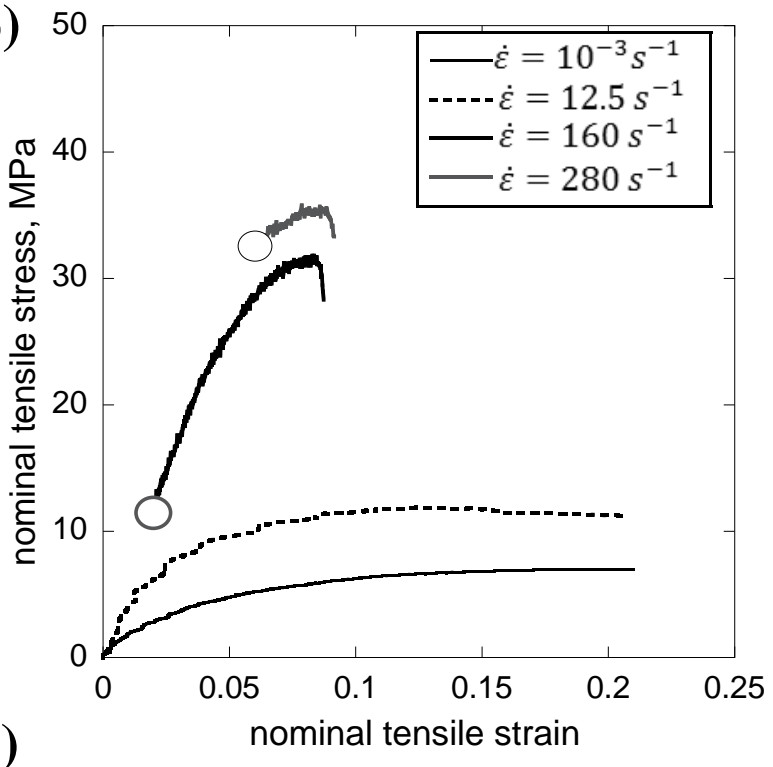

(c)

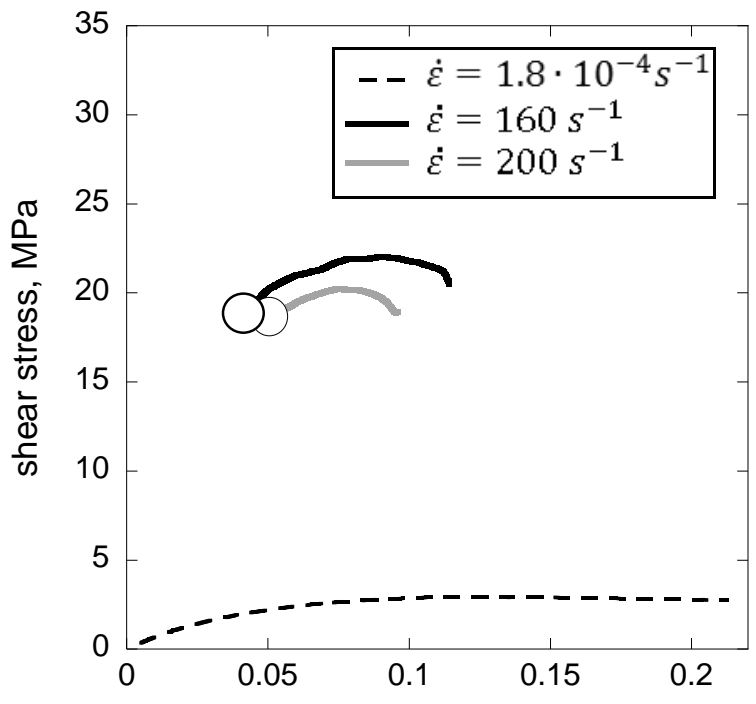

shear strain

Fig 6. Stress versus strain response of the foam at different strain rates in compression (a), tension (b) and shear (c). A high sensitivity to strain rate is displayed in each loading condition. 


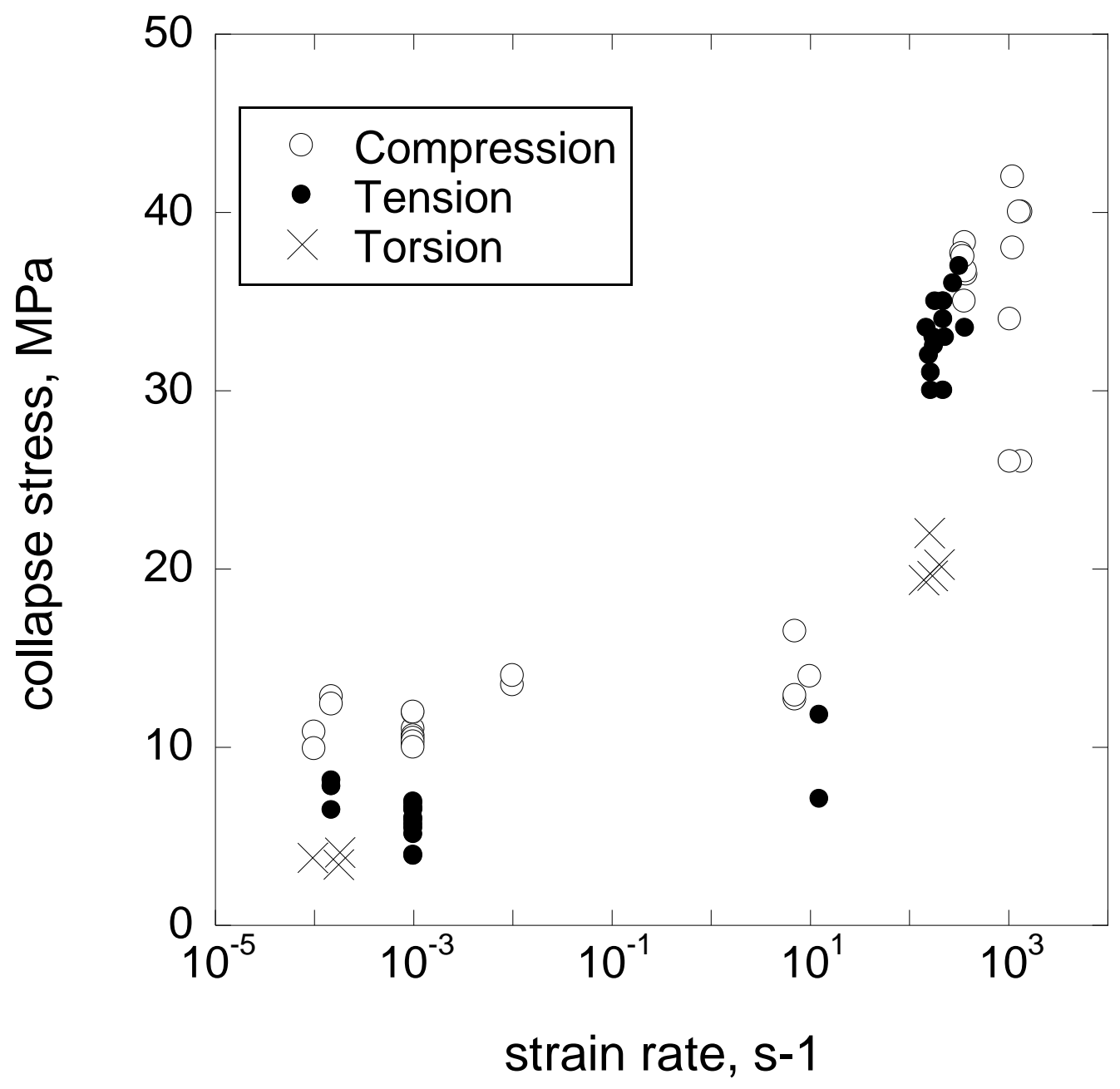

Fig.7. Rate dependence of measured plateau stress (in compression) and peak stress (in tension and torsion). 


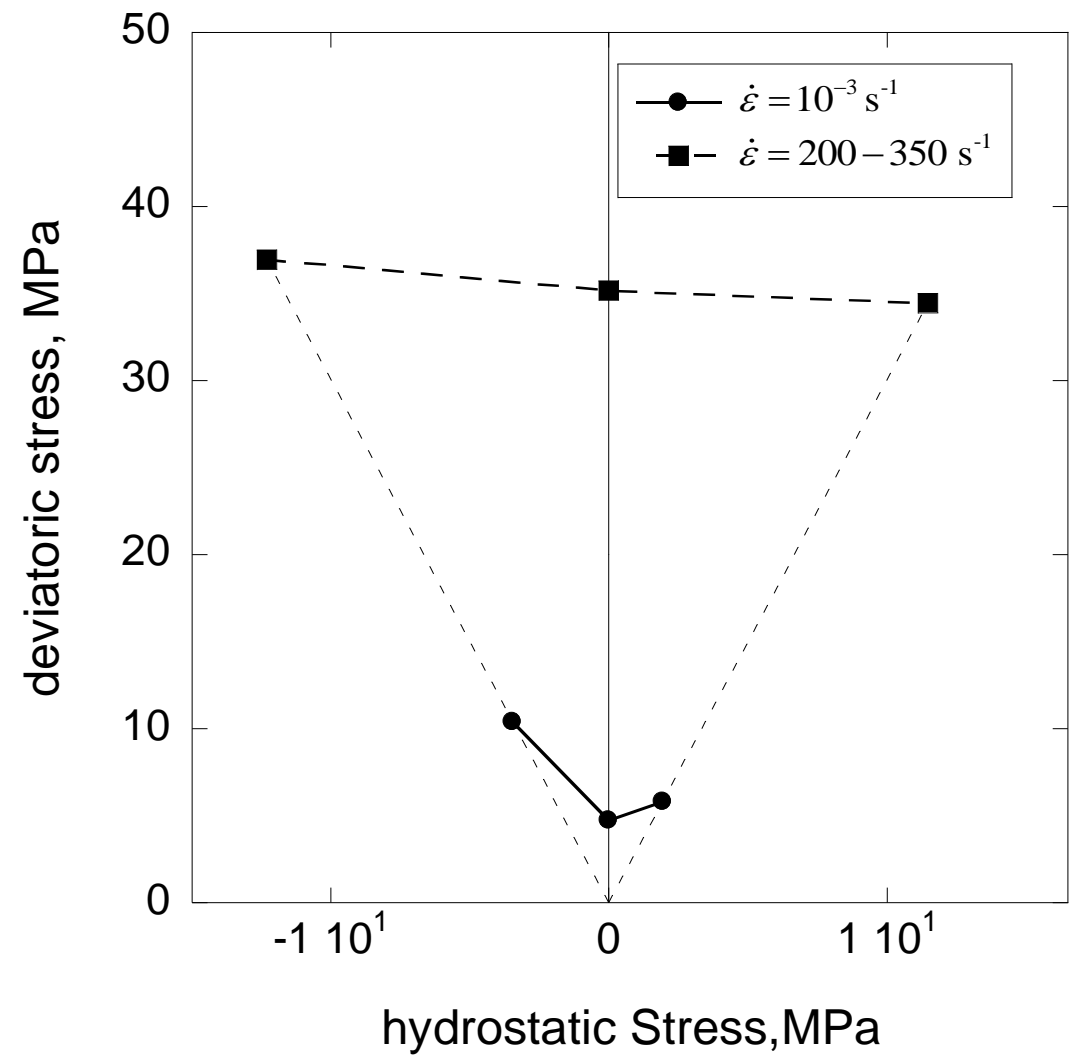

Fig. 8. Partial failure envelope for the syntactic foam at low and high strain rate. Stress values refer to the compressive collapse stress and the peak stress in tension and shear. Circles refer to low strain rate, squares to high strain rate as indicated. 
- Response of a syintactic foam measured in compression, tension and shear at both quasi-static and high rates of strain.

- The PU syntactic foam displays a strain rate sensitivity superior to that of solid PU and of conventional PU foams of similar density

- The foam displays great tensile and shear ductility at low strain rates and partially retains this at high strain rates.

- The foam displays a tension/compression asymmetry in the quasi-static regime but this is lost at high rates of strain. 